Enzymsüße

\title{
Multi-Enzymsystem zur Herstellung eines alternativen Zuckersirups
}

\author{
SABINE-LUTZ-WAHL ${ }^{1}$, EVA PROSS ${ }^{1}$, JÖRG HINRICHS ${ }^{2}$, LUTZ FISCHER ${ }^{1}$ \\ ${ }^{1}$ FACHGEBIET BIOTECHNOLOGIE UND ENZYMWISSENSCHAFT, INSTITUT FÜR LEBENS- \\ MITTELWISSENSCHAFT UND BIOTECHNOLOGIE, UNIVERSITÄT HOHENHEIM \\ ${ }^{2}$ FACHGEBIET MILCHWISSENSCHAFT UND -TECHNOLOGIE, INSTITUT FÜR LEBENS- \\ MITTELWISSENSCHAFT UND BIOTECHNOLOGIE, UNIVERSITÄT HOHENHEIM
}

\section{Energy-rich foods lead to an increase of overweight people in our societies. Mainly the fat and sugar content in foods is responsible for this progress. However, both components are very important for the taste and acceptance of foods. Thus, the abundant available lactose will be enzymatically modified by using the enzymes $\beta$-galactosidase, $\mathrm{L}$-arabinose isomerase and glucose isomerase in order to generate a new natural sugar syrup. The new sugar mixture will be much sweeter than lactose.}

DOI: $10.1007 / \mathrm{s} 12268-020-1467-2$

(C) Die Autoren 2020

Zuckerhaltige, süßschmeckende Lebensmittel erfreuen sich einer ungebremst starken Nachfrage bei den Konsumenten, obwohl seit langem bekannt ist, dass eine hohe Energiezufuhr durch Lebensmittel bei gleichzeitig geringer körperlicher Bewegung zu einer Vielzahl von gesundheitlichen Problemen führen kann, wie Bluthochdruck, Typ-2-Diabetes, Herz-Kreislauf-Erkrankungen und Übergewicht [1]. Deshalb wird seit Jahrzehn-

ten nach und an Zuckeraustauschstoffen und Süßstoffen geforscht, die bei gleicher Süße einen geringen physiologischen Energiegehalt besitzen. Die im Markt befindlichen Süßungsmittel sind im Vergleich zu den natürlichen Zuckern wie Saccharose (allg. Haushaltszucker) oder Glucose (Traubenzucker) jedoch nicht gleich in der geschmacklichen Wahrnehmung. Außerdem ist für Zuckeraustauschstoffe und Süßstoffe die

Tab. 1: Süße und Energiegehalt verschiedener Zucker sowie Abschätzung des Einsparungspotenzials für ein Milcherzeugnis pro $100 \mathrm{~g}$ auf Basis der Daten von [18].

\begin{tabular}{|c|c|c|c|c|}
\hline & $\begin{array}{l}\text { Relative } \\
\text { Süße }{ }^{1)} \text { (RS) }\end{array}$ & $\begin{array}{l}\text { Energie } \\
\text { in } \mathrm{kJ} / \mathrm{g}\end{array}$ & $\begin{array}{l}\text { Milcherzeugnis } \\
+ \text { Zucker, z. B. } \\
\text { Fruchtjoghurt }\end{array}$ & $\begin{array}{l}\text { Lactosered. } \\
\text { Milcherzeugnis } \\
+ \text { tri-enzym. Sirup }\end{array}$ \\
\hline Lactose & 16 & 16,8 & $4,6 \mathrm{~g}$ & - \\
\hline Galactose & 32 & 16,8 & - & $2,3 \mathrm{~g}$ \\
\hline Glucose & 74 & 16,8 & - & $2,3 \mathrm{~g}$ \\
\hline Tagatose & 92 & $6,3^{3)} / 12,6^{4)}$ & - & - \\
\hline Fructose & 172 & 16,8 & - & - \\
\hline$S \ddot{A}^{2)}$ im Milcherzeugnis & - & - & 74 & 244 \\
\hline Zugabe Tri-enzym. Sirup & 84 & 15,1 & - & $4,0 \mathrm{~g}$ \\
\hline Zugabe Saccharose & 100 & 16,8 & $5,0 \mathrm{~g}$ & \\
\hline $\begin{array}{l}\text { SÄ im Milcherzeugnis } \\
+ \text { Zugabe }\end{array}$ & - & - & 574 & 574 \\
\hline \multicolumn{3}{|l|}{ Sacharosereduktion in \% } & - & 100 \\
\hline \multicolumn{3}{|c|}{ Energie aus Zucker kJ/100 g (Reduktion) } & $159(0 \%)$ & $138(13 \%)$ \\
\hline
\end{tabular}

Zusatzstoff-Zulassungsverordnung zu beachten und eine Deklaration ist vorgeschrieben, was zusätzlich für Skepsis beim Konsumenten sorgt.

Interessant scheint daher die enzymatische Umwandlung eines natürlichen Zuckers, wie der Lactose. Zwar hat Lactose im Vergleich zu Saccharose eine wesentlich geringere Süße von nur ca. 16 Prozent, sie fällt jedoch in sehr großen Mengen als Nebenstrom (Süß- oder Sauermolke, ca. pH = 6,7 oder $\mathrm{pH} \leq 5,2)$ bei der Käseherstellung in den Molkereien an und wird momentan nur teilweise einer weiteren Wertschöpfung zugeführt. Durch moderne Membranverfahren, wie sie heute in den Molkereien ohnehin bereits verwendet werden, könnten diese Molkenebenströme in ihrer Zusammensetzung für einen enzymatischen Prozess maßgeschneidert werden. Ziel ist es hierbei, die Lactose- und Salzkonzentrationen so einzustellen, dass der anschließende tri-enzymatische Umsetzungsprozess der Lactose optimal abläuft. Der tri-enzymatische Prozessschritt soll die Lactose dann vollständig in ein Monosaccharidgemisch umwandeln. Dieses Monosaccharidgemisch stellt einen „Zuckersirup“ aus Galactose, Glucose, Fructose und Tagatose dar, der eine natürliche Süße besitzt und alternativ zur Saccharose, z. B. in der Herstellung von süßen Backwaren oder Milchprodukten verwendet werden kann. Die Menge an Zuckersirup, die für die Einstellung der gleichen Süße im Lebensmittel wie mit Saccharose notwendig wäre, besitzt einen geringeren physiologischen Energiegehalt und sorgt somit für eine um bis zu 13 Prozent verminderte Energiezufuhr (Tab. 1).

Als Bezugsgröße für die Süßkraft von Zuckern in Lebensmitteln wird die relative Süße (RS) einer 10\%igen (w/v) Zuckerlösung im Vergleich zu einer 10\%igen Saccharoselösung (w/v), die als Referenz dient und einen fixen RS-Wert von 100 bekommt, verwendet. Lactose weist, wie oben bereits angesprochen, lediglich eine relative Süße von 16 auf, jedoch kann bereits durch die Lactosehydrolyse die Süßkraft von ca. 20 Prozent auf ca. 70 Prozent gesteigert werden [2]. Die 
Hydrolyse von Lactose erfolgt enzymatisch mittels $\beta$-Galactosidasen (EC 3.2.1.23), wodurch theoretisch äquimolar die Monosaccharide Glucose $(\mathrm{RS}=74)$ und Galactose $(\mathrm{RS}=32$ ) entstehen (Abb. 1, [3]). Eine zusätzliche Steigerung dieser Süßkraft wird dann durch eine zweite enzymatische Reaktion möglich: Mit L-Arabinoseisomerasen (EC 5.3.1.4) wird Galactose teilweise in das Monosaccharid Tagatose ( $\mathrm{RS}=92)$ isomerisiert (Abb. 1, [4]). Wie viel Tagatose entsteht, ist von der Gleichgewichtslage der Reaktion abhängig, also der Konzentration der Substanzen, der Temperatur, dem Druck und dem pH-Wert. In einem bi-enzymatischen Prozess, der die Enzyme $\beta$-Galactosidase und L-Arabinoseisomerase nutzt, entsteht somit ein Gemisch der Monosaccharide Glucose, Galactose und Tagatose (Abb. 1).

Die Lactosehydrolyse mittels $\beta$-Galactosidasen ist eine etablierte Technologie, die bereits seit den 1970er-Jahren genutzt wird, um lactosefreie Milchprodukte für Personen mit Lactoseintoleranz zu produzieren $[3,5$, 6]. Die industrielle Herstellung der $\beta$-Galactosidasen erfolgt mittels Mikroorganismen [7], entweder mit Aspergillus, Kluyveromyces oder Bacillus [8]. Die enzymatische Lactosehydrolyse erfolgt z. B. bei H-Milch bei unter $10{ }^{\circ} \mathrm{C}$ innerhalb von max. $48 \mathrm{~h}$ vor der Ultrahocherhitzung oder sie kann sogar durch sterile Zugabe der $\beta$-Galactosidasen direkt in der Milchverpackung beim Transport realisiert werden. In jedem Fall wird die Lactose nahezu vollständig gespalten $(<0,1 \% \mathrm{w} / \mathrm{v})$.

Im angestrebten bi-enzymatischen Prozess soll die entstandene Galactose mittels L-Arabinoseisomerasen (L-AI) partiell zu Tagatose isomerisiert werden. Für diese Reaktion steht aktuell kein kommerziell erhältliches Enzympräparat zur Verfügung. Die in der Literatur beschriebenen L-AI kommen aufgrund ihrer biochemischen Eigenschaften eher nicht für den Einsatz in einem multienzymatischen Verfahren mit membrantechnologisch gewonnenen Lactoselösungen in Betracht [9]. Daher wird in einem gerade begonnenen FEI-Projekt (AiF 21100 N „Sirup aus Lactose-haltigen Nebenströmen“) nach neuen mikrobiellen L-AI gesucht, die bessere Eigenschaften bezüglich Aktivität und Stabilität besitzen. Für die Identifizierung neuer L-AI werden mehrere Methoden angewandt (Abb. 2). Zum einen wird in metagenomischer DNA aus unterschiedlichen Umweltproben mittels degenerierter Primer, die komplementär zu hochkonservierten Regionen von L-AI sind, nach neuen Genen von

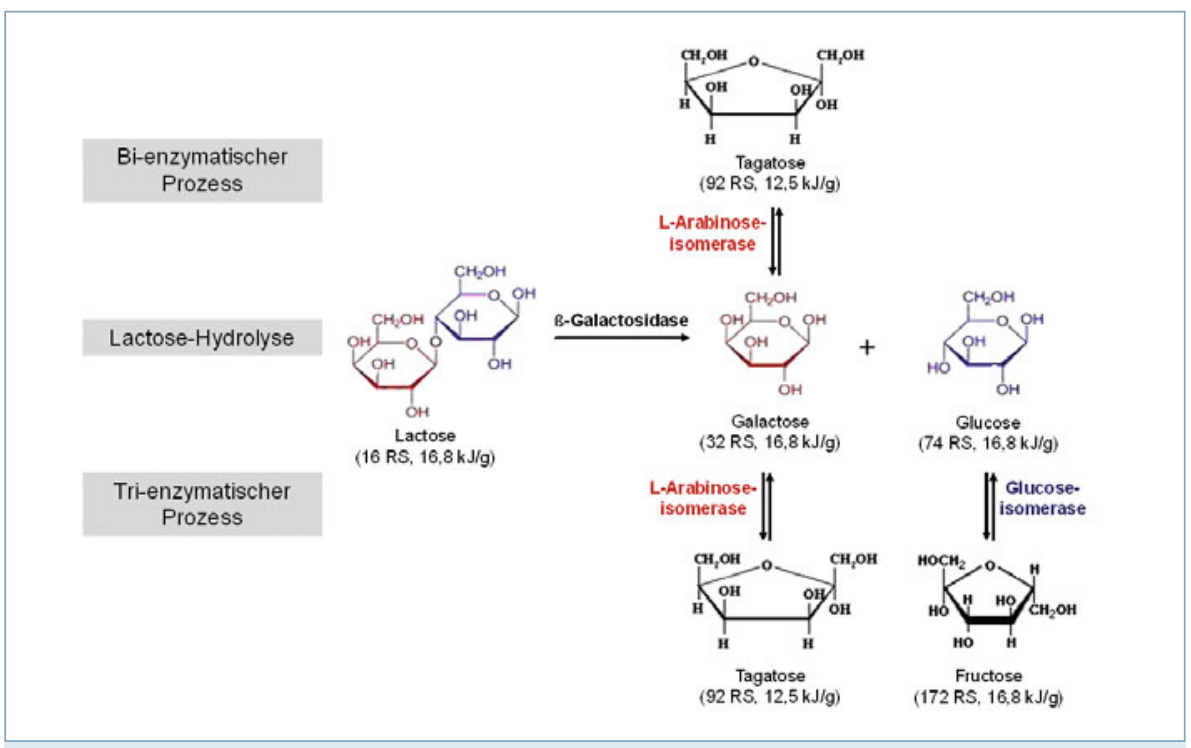

A Abb. 1: Bi- und tri-enzymatischer Schritt zur Generierung eines Zuckersirups aus Lactose. In einem bi-enzymatischen Schritt mit den Enzymen $\beta$-Galactosidase und L-Arabinoseisomerase entsteht ein Zuckergemisch aus den Monosacchariden Galactose, Glucose und Tagatose. In einem tri-enzymatischen Prozess kann dieses Zuckergemisch mittels Glucose-Isomerasen um das Monosaccharid Fructose erweitert werden [18].

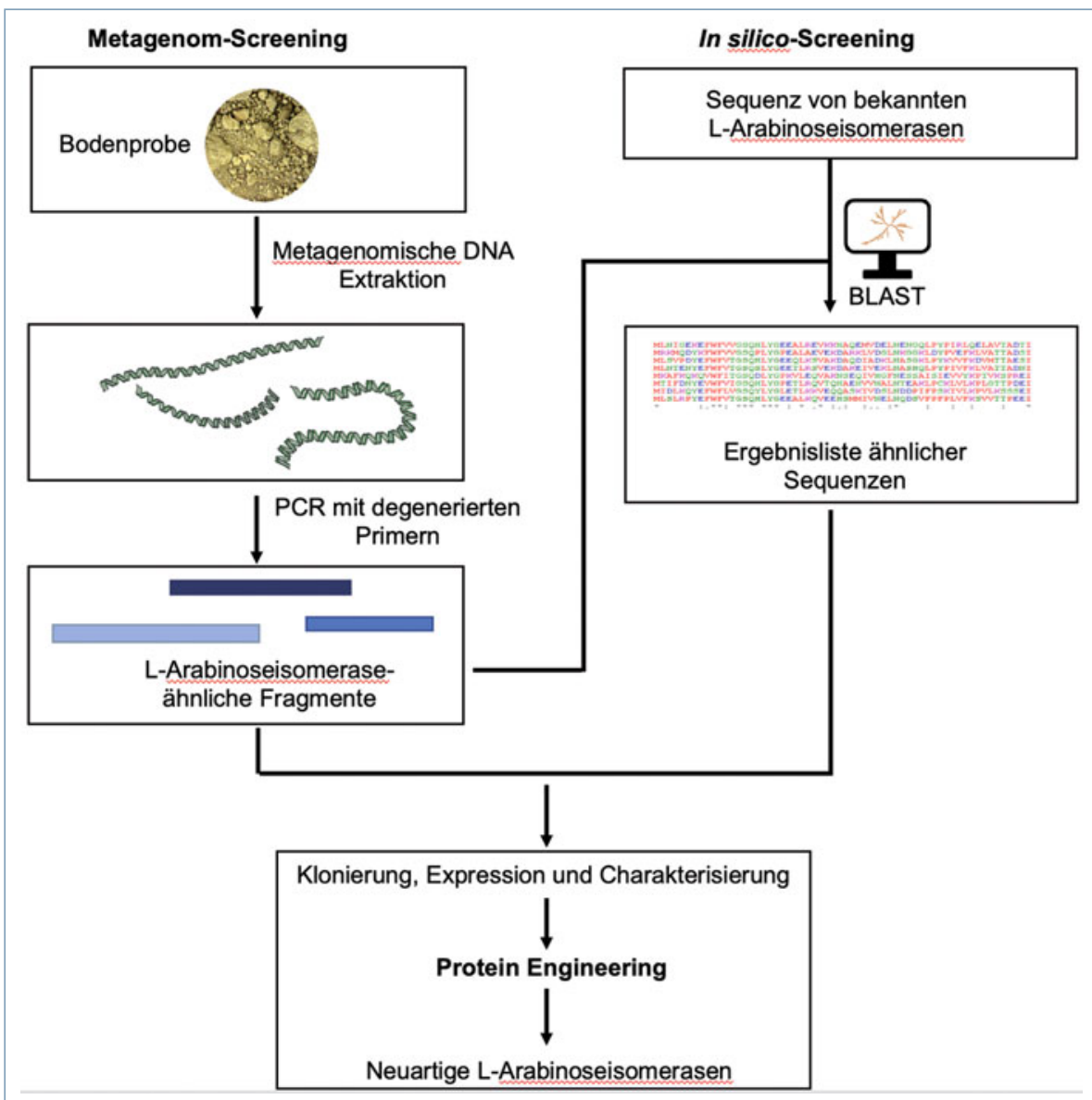

$\Delta$ Abb. 2: Metagenom- und in silico-Screening zur Identifizierung neuer L-Arabinoseisomerasen für ein multi-enzymatisches System zur Zuckersirup-Herstellung. Die putativen L-Arabinoseisomerasen werden zunächst in Escherichia coli kloniert, exprimiert und anschließend charakterisiert. Über protein engineering sollen die biochemischen Eigenschaften optimiert werden. 
L-AI gesucht [10]. Zusätzlich wird ein in silicoScreening mithilfe der Aminosäuresequenzen von bereits bekannten L-AI mittels BLAST und Proteindatenbanken durchgeführt $[10,11]$. Die putativen L-AI können anschließend zunächst rekombinant in Escherichia coli hergestellt und für den Zuckersirup-Prozess charakterisiert werden. Ziel dieses Teils der Forschungsarbeiten ist es, eine mikrobielle L-AI zu identifizieren, die eine hohe spezifische Aktivität bei einem $\mathrm{pH}$ Wert zwischen 4,3 bis 6,7 und einem Temperaturbereich von $5-30{ }^{\circ} \mathrm{C}$ aufweist. Zudem benötigen die neuen mikrobiellen L-AI einen niedrigen $\mathrm{K}_{\mathrm{m}}$-Wert $(\leq 10 \mathrm{mM})$ für das Substrat Galactose, um eine effiziente Isomerisierung von Galactose in Tagatose zu realisieren. Als dritte Methode zur Identifizierung einer geeigneten L-AI wird protein engineering durch gezielte Mutagenese durchgeführt, um die Eigenschaften der L-Arabinoseisomerasen an die prozessrelevanten Bedingungen anzupassen [12].

Der Vorteil des bi-enzymatisch generierten Zuckersirups ist ein verminderter physiologischer Brennwert durch die teilweise Isomerisierung von Galactose zu Tagatose. Im Vergleich zum Energiegehalt von Lactose, Glucose und Galactose mit 16,8 kJ/g weist Tagatose nämlich einen geringeren Energiegehalt von 6,3 kJ/g [13] bzw. 12,5 kJ/g [14] auf.

Um jedoch eine vergleichbare Süße in Lebensmitteln wie mit Saccharose zu erlangen, ist ein weiterer enzymatischer Schritt zur Umwandlung von Glucose (RS = 74) in die wesentlich süßere Fructose $(\mathrm{RS}=172)$ erforderlich. Dies geschieht durch die Glucose-Isomerase (GI, EC 5.3.1.5), die die reversible Isomerisierung von Glucose zu Fructose katalysiert. GI ist ein wichtiges kommerzielles Enzym für die Herstellung von high fructose corn syrup (HFCS) und wird bereits seit den 1970er-Jahren immobilisiert in der Industrie verwendet $[15,16]$. HFCS kommt beispielsweise in vielen Erfrischungsgetränken und anderen Lebensmittelprodukten vor, wo es den Rüben- oder Rohrzucker ersetzt [17].

Der in dem FEI-Forschungsprojekt angestrebte Zuckersirup-Prozess benötigt somit drei aufeinander abgestimmte Enzyme: $\beta$-Galactosidase, L-Arabinoseisomerase (L-AI) und Glucoseisomerase (GI). Diese drei Enzymklassen wurden bereits in immobilisierter Form in einem enzymatischen Prozess mit Lactose als Ausgangssubstrat getestet und sowohl sequenziell als auch gleichzeitig eingesetzt [24]. Durch Ko-Immobilisierung der Enzyme konnte die Lactose in einer demineralisierten Mozzarella-Molke vollständig zu Galactose und Glucose hydrolysiert und eine Isomerisierung dieser Monosaccharide zu 45 Prozent Tagatose und 39 Prozent Fructose bei $50{ }^{\circ} \mathrm{C}$ und $\mathrm{pH} 7$ erreicht werden. Allerdings zeigte in diesem Fall die L-AI eine geringe Prozessstabilität. Bei Durchführung des Prozesses bei $30{ }^{\circ} \mathrm{C}$ sanken alle Umsetzungsraten [18].

Im Rahmen dieses FEI-Projekts soll nun, ausgehend von den eingangs genannten und durch Membrantechnik maßgeschneiderten lactosehaltigen Nebenströmen der Milchindustrie, bi- und tri-enzymatische Prozesse zur Herstellung eines Zuckersirups mit freien Enzymen etabliert werden. Die wissenschaftliche Herausforderung besteht hierbei den Prozess mit drei löslichen, nicht-immobilisierten Enzymen unter technisch relevanten Prozessbedingungen durchzuführen. Der Prozess soll bei niedrigen Temperaturen $\left(5-30{ }^{\circ} \mathrm{C}\right)$ erfolgen. Für die Umsetzung von Lactose zu Glucose und Galactose bzw. zur weiteren Umsetzung von Glucose zu Fructose werden kommerziell verfügbare Enzympräparate eingesetzt. Für die Umsetzung von Galactose zu Tagatose wird, wie oben bereits beschrieben, nach einer geeigneten neuen L-Arabinoseisomerase gescreent.

\section{Literatur}

[1] Grembecka M (2015) Sugar alcohols-their role in the modern world of sweeteners. Eur Food Res Technol 241:1-14 [2] Novalin S, Neuhaus W, Kulbe KD (2005) A new innovative process to produce lactose-reduced skim milk. J Biotechnol 119:212-218

[3] Husain Q (2010) Beta galactosidases and their potential applications: a review. Crit Rev Biotechnol 30:41-62

[4] Cheetham PSJ, Wootton AN (1993) Bioconversion of D-galactose into D-tagatose. Enzyme Microb Tech 15:105-108 [5] Choi SH, Lee SB, Won HR (2007) Development of lactose-hydrolyzed milk with low sweetness using nanofiltration. Asian Australas J Anim Sci 20:989-993

[6] Somkutl GA, Holsinger VH (1997) Microbial technologies in the production of low-lactose dairy foods/Technologías microbiológicas para la elaboración de productos lácteos con bajo contenido en lactosa. Food Sci Technol Int 3:163-169 [7] Mahoney RR (1997) Lactose: enzymatic modification. In Fox PF (Hrsg.). Advanced dairy chemistry. Chapman \& Hall, New York, 77-126

[8] Zolnere K, Ciprovica I (2017) The comparison of commercially available $\beta$-galactosidases for dairy industry: review. Res Rural Dev 1:215-222

[9] Roy S, Chikkerur J, Roy SC et al. (2018) Tagatose as a potential nutraceutical: production, properties, biological roles, and applications. J Food Sci 83:2699-2709

[10] Cai X, Seitl I, Mu W et al. (2018) Combination of sequence-based and in silico screening to identify novel trehalose synthases. Enzyme Microb Tech 115:62-72 [11] Krewinkel M, Kaiser J, Merz M et al. (2015) Novel cellobiose 2-epimerases for the production of epilactose from milk ultrafiltrate containing lactose. J Dairy Sci 98:3665-3678 [12] Laksmi FA, Arai S, Tsurumaru H et al. (2018) Improved substrate specificity for D-galactose of L-arabinose isomerase for industrial application. BBA Proteins Proteom 1866:10841091

[13] Levin GV (2002) Tagatose, the new GRAS sweetener and health product. J Med Food 5:23-36

[14] Turck D, Bresson JL, Burlingame B et al. (2016)

Scientific opinion on the energy conversion factor of

D-tagatose for labelling purposes. EFS2 14:209
[15] Bhosale SH, Rao MB, Deshpande VV (1996) Molecular and industrial aspects of glucose isomerase. Microbiol Rev 60:280-300

[16] Liu, ZQ, Zheng W, Huang JF et al. (2015) Improvement and characterization of a hyperthermophilic glucose isomerase from Thermoanaerobacter ethanolicus and its application in production of high fructose corn syrup. J Ind Microbiol Biot 42:1091-1103

[17] Parker K, Salas M, Nwosu VC (2011) High fructose corn syrup: production, uses and public health concerns.

Biotechnol Mol Biol Rev 5:71-78

[18] Torres P, Batista-Viera F (2017) Immobilized trienzymatic system with enhanced stabilization for the biotransformation of lactose. Molecules 22:284

Funding Open Access funding enabled and organized by Projekt DEAL. Open Access Dieser Artikel wird unter der Creative Commons Namensnennun 4.0 International Lizenz veröffentlicht, welche die Nutzung, Vervielfältigung, Bearbeitung, Verbreitung und Wiedergabe in jeglichem Medium und Forma erlaubt, sofern Sie den/die ursprünglichen Autor(en) und die Quelle
ordnungsgemäß nennen, einen Link zur Creative Commons Lizenz beifügen und ordnungsgemäß nennen, einen Link zur Creative Commons Lizenz beifü
angeben, ob Änderungen vorgenommen wurden. Die in diesem Artikel enthaltenen Bilder und sonstiges Drittmaterial unterliegen ebenfalls der genannten Creative Commons Lizenz, sofern sich aus der Abbildungslegen nichts anderes ergibt. Sofern das betreffende Material nicht unter der genannten Creative Commons Lizenz steht und die betreffende Handlung nicht nach gesetzlichen Vorschriften erlaubt ist, ist für die oben aufgeführten Weiterverwendungen des Materials die Einwilligung des jeweiligen
Rechteinhabers einzuholen. Weitere Details zur Lizenz entnehmen Sie bitte der Lizenzinformation auf http://creativecommons.org/licenses/by/4.0/deed.de.
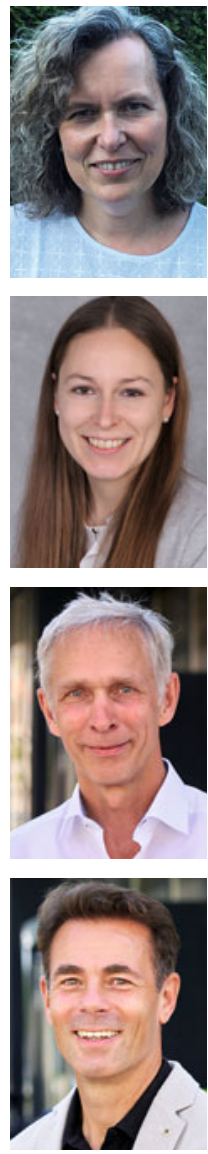

Sabine-Lutz-Wahl, Eva Pross, Jörg Hinrichs und Lutz Fischer (von oben nach unten)

Korrespondenzadresse:

Prof. Dr. Lutz Fischer

Fachgebiet Biotechnologie und

Enzymwissenschaft

Institut für Lebensmittelwissenschaft und

Biotechnologie

Universität Hohenheim

Garbenstraße 25

D-70599 Stuttgart

Lutz.Fischer@uni-hohenheim.de 